\title{
Economic Evaluation of Social Care Interventions: Lessons Drawn from a Systematic Review of the Methods Used to Evaluate Reablement
}

\author{
Rita Faria ${ }^{{ }^{*}}$, Noemi Kiss ${ }^{2}$, Fiona Aspinal ${ }^{3}$, Melissa Harden ${ }^{4}$ and Helen Weatherly ${ }^{1}$ \\ ${ }^{1}$ Centre for Health Economics, University of York, York, UK \\ ${ }^{2}$ Department of Health Economics, Centre for Public Health, Medical University of Vienna, Austria \\ ${ }^{3}$ Social Policy Research Unit, University of York, York, UK \\ ${ }^{4}$ Centre for Reviews and Disseminations, University of York, York, UK
}

\begin{abstract}
Reablement helps individuals to regain or retain the ability to live independently after an injury and/or exacerbation of an illness. Despite the lack of clear evidence on its effectiveness and cost-effectiveness, or on the optimal model for its implementation, reablement is hailed as one solution to the increased demand for long term care. This study systematically reviews, synthesises and critically appraises the economic evidence on reablement, and makes recommendations to improve future research. A total of 13 studies were included. Data were extracted on: decision, context, study design, intervention, comparators, effectiveness, outcomes, resource use and costs, analytic methods, and study findings. Reablement has been shown to be cost-effective; however the reporting on the decision the study aims to inform and the context could typically be improved and there is uncertainty on the costs and benefits of reablement in the longer term, between different models of service delivery and the impact on carers. All studies used only the data on outcomes and costs that was collected within the study and evaluated cost-effectiveness over the data collection follow-up, but this approach was not justified. These methods results should inform future research. Economic evaluations on reablement should: (i) include all available evidence on the relevant interventions and comparators over the appropriate time horizon, (ii) compare the benefits of the intervention with its opportunity costs and (iii) consider subgroup analysis to identify the groups of individuals most likely to benefit from reablement and therefore better target interventions and resources. More methodological research and guidance is needed on standardised outcome measures on general wellbeing, on informal carer's burden and on how to trade-off the costs and benefits in different sectors. These recommendations can help improve quality and relevance for decision makers in the future and to guide future economic evaluations and methodological research.
\end{abstract}

Keywords: Reablement; Systematic review; Economic evaluation; Complex intervention/s

\section{Introduction}

Reablement, also termed restorative care, is a time limited intervention to help individuals regain or retain the ability to live independently after an injury or illness [1]. Reablement is a complex intervention [2]. It is a flexible service tailored around the individual's needs and adapted to their progress and feedback. It involves several interactive components, from the assessment that determines the initial service package, to the different professionals involved in its delivery (e.g., reablement worker, occupational therapist, physiotherapist, nurse, social worker) and the different activities (e.g., shopping, preparation of meals, self-care) that constitute the person's reablement intervention. The outcomes achieved by reablement depend on the individual's engagement and their rapport with the reablement team. Lastly, the outcomes achieved by reablement can vary and are individual-specific. For example, these outcomes may be functional in nature, such as being able to dress and wash independently, or related to community engagement such as accessing social activities outside the home.

Although reablement has been hailed as an effective and costeffective intervention, there is currently little guidance on its implementation $[3,4]$. A growing number of countries across the world are investing in reablement to reduce long-term care costs and improve wellbeing of their dependent population [5-9]. The UK, for example, has made $£ 300$ million available per year over $2012-15$ to fund reablement services [10]. This public investment may not be used to its maximum benefit given the lack of clear evidence on reablement.

Economic evaluation can help ensure that public investment in reablement achieves the maximum return. However, the complexity of reablement makes it difficult to evaluate. Current methodological recommendations on complex interventions lack detail on how to ensure that results are informative for decision making [2]. This study reviews the economic evaluation evidence on reablement to understand the evaluation methods used and to draw lessons on how to improve its evaluation in future research. This study is part of a larger project on the effectiveness and cost-effectiveness of different service models of reablement delivered in the UK. The conclusions of this review will inform the methods for economic evaluation within this wider study to ensure the conclusions are useful for decision makers and to help inform the implementation of reablement in practice.

\section{Materials and Method}

The systematic review examined two key questions: (i) how has the cost-effectiveness of reablement been evaluated? (ii) what could be done better? The systematic review methods were informed by the Centre for Reviews and Dissemination (CRD) guidelines and the Social

*Corresponding author: Rita Faria, Centre for Health Economics, University of York, Heslington, York, YO10 5DD, UK, Tel: 019043214 35; Fax: 01904 3214 02; E-mail: rita.nevesdefaria@york.ac.uk

Received November 30, 2015; Accepted January 03, 2016 Published January 10, 2016

Citation: Faria R, Kiss N, Aspinal F, Harden M, Weatherly H (2016) Economic Evaluation of Social Care Interventions: Lessons Drawn from a Systematic Review of the Methods Used to Evaluate Reablement. Health Econ Outcome Res Open Access 2: 107.

Copyright: ( 2015 Faria R, et al. This is an open-access article distributed under the terms of the Creative Commons Attribution License, which permits unrestricted use, distribution, and reproduction in any medium, provided the original author and source are credited. 
Care Institute for Excellence (SCIE) guidelines [11,12] on conducting systematic reviews. The review was conducted between November 2014-November 2015 and the aim was to inform the data collection and economic evaluation methodology used in subsequent phases of the wider study.

\section{Search strategy}

The literature searches aimed to systematically identify relevant peer-reviewed and grey literature reporting economic evaluations of reablement. Databases covering health, social care, economics and social science were searched in January-February 2015: MEDLINE, MEDLINE in-Process, ASSIA, EconLit, Health Management Information Consortium (HMIC), NHS Economic Evaluations Database (NHS EED), and Social Care Online. The search terms included a range of text words, synonyms and subject headings related to reablement combined with a study design search filter to limit retrieval to economic studies [13]. In addition, discussions were held with experts within the wider project team to identify additional publications. The Supplementary Appendix details the search strategies.

\section{Study selection criteria}

Screening: Three levels of screening were implemented: i) title screening, (ii) abstract screening and (iii) full text screening by three team members working independently. Disagreements were resolved through discussion with another member of the team.

Inclusion and exclusion criteria: The inclusion and exclusion criteria were intended to identify papers on reablement. An intervention was considered to be reablement if it was a personalised, time-limited (up to 12 weeks), goal-oriented intervention, which focussed on restoring or maintaining function and/or managing everyday activities at home, delivered in the individual's usual place of residence. The target population was adults who cannot manage independently but who have the potential of re-learning skills or learning techniques to help cope with their condition. To be included, studies were required to compare at reablement with another intervention, such as no reablement, home-care or rehabilitation at the hospital or clinic. Full economic evaluations were included namely cost-benefit, cost-consequence, cost-effectiveness and cost-utility evaluations. Cost-minimisation studies were included if the original intention of the authors had been to conduct a cost-effectiveness study but no difference in effectiveness was found. Studies evaluating interventions in children and adolescents, in people with drug misuse problems, in people with psychiatric conditions or in people at the end of life were excluded because these are not the target population for generic reablement services in the UK.

\section{Data extraction}

The aim of the data extraction was to understand the data and methods used in previous studies. Table 1 show the variables included in the data extraction form. When studies reported only the costs, the companion study on the effectiveness outcomes was consulted to complete the data extraction. Data were categorised under topics and extracted on:

- Research question: the objectives and the perspective of the analysis.

- Intervention (i.e., reablement): objective, how it was described and the skill-mix of the team to understand the heterogeneity of interventions with a reabling approach and, where possible, tease out which components drive outcomes and costs.
- $\quad$ Comparators (i.e., interventions compared with reablement): description. Fewer details were extracted on the comparators because this review aimed to aid understanding of the methods used for economic evaluation of these types of services rather than to determine the cost-effectiveness of reablement.

- Context: country and publication date to provide insight into the context of reablement and its generalisability to the current UK context.

- Design: type of economic evaluation, time horizon and analytic approach used (regression analysis, decision modelling).

- $\quad$ Effectiveness data: study design, sample size and assumptions required to use the data.

- $\quad$ Outcome measures: which outcome measures were collected and in whom (i.e., service user or carer).

- $\quad$ Resource use and costs: type of costs included.

- Analytic methods: how the study concluded on the costeffectiveness of reablement (the assessment of cost-effectiveness), sensitivity analysis, subgroup analysis.

- Findings: study's conclusions and methodological uncertainties and areas for future research identified, as these have direct implications for learning that can be taken from the studies.

\section{Analysis and synthesis of the results}

Data were analysed in tabulated and graphical form. A narrative analysis was undertaken to understand the methods and data used, and to suggest areas for improvement.

\section{Results}

Figure 1 presents the PRISMA flowchart of the study selection process. Thirteen studies were included in the review. Full data extraction tables are available from the authors on request.

\section{Research question}

Table 2 summarises the research question. The studies aimed to compare the costs and outcomes of reablement in patients after stroke, in elderly patients or for anyone referred. The perspective of the analysis was stated in eight studies: health care and private [14], health and social care [15], societal [16,17], health and social care [18-20] and health social care and private [21]. In the other five studies, the perspective inferred from the costs included in the analysis was health [22], social care [23], health and social care [24,25], and health, social care and private [26]

\section{Intervention}

The studies used different names for the intervention: seven studies used the term 'rehabilitation' in the intervention's name: 'home-based rehabilitation' [16,24,26,27], 'early discharge and rehabilitation service' [15], 'home rehabilitation' [26], 'domiciliary rehabilitation' [17,18]. Two studies used the term 'reablement' $[20,23]$. The term 'restorative' $[21,25]$ was used twice and 'transitional' was used once [19].

Of the 13 included studies, all but Parker et al stated the objectives of the intervention(s) [16]. The most frequent objective was to reduce institutionalisation, including admissions and readmissions to hospital, shorten hospital stay and delay admission to care home $(\mathrm{N}=7)$ $[15,17,19-22,26]$. Five studies mentioned that an improvement in health and functional status $[14,17,19,24,26]$, and five aimed to achieve 

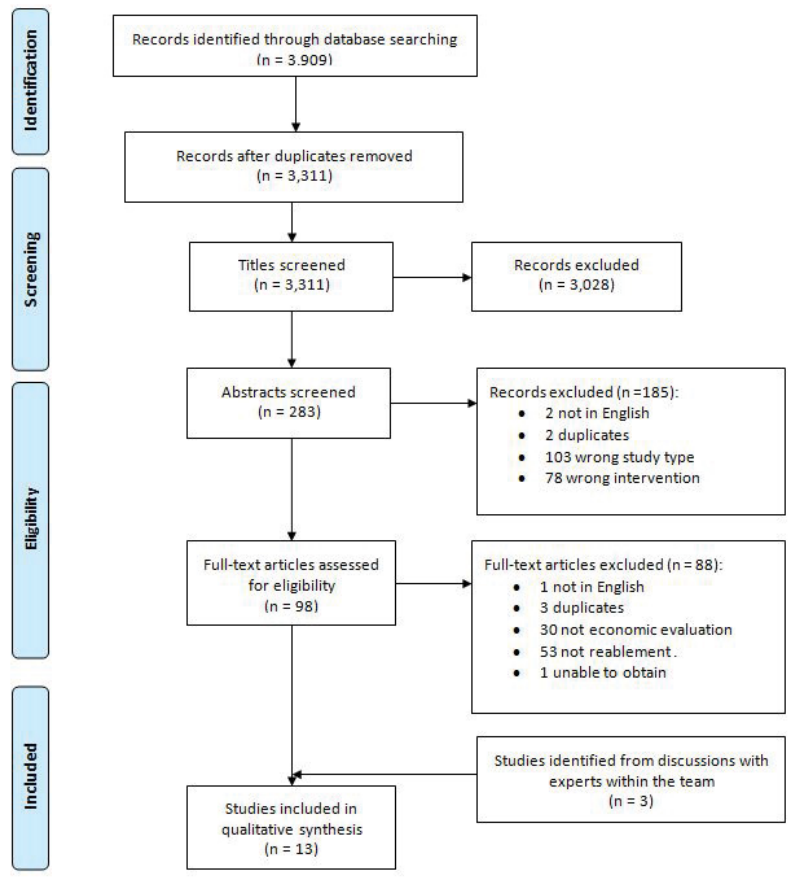

Figure 1: PRISMA diagram.

cost savings $[17,18,20,24,26]$. Interventions that aimed to achieve cost savings sought to do so in terms of lower service use in the long-term [20], reduction in hospital admissions $[17,26]$ and initial length of stay $[17,24,26]$ or a reduction in day-hospital usage [18]. To help people build confidence and/or independence was mentioned by four studies $[17,20,23,25]$. Other objectives referred to were to facilitate integration in the community [14], to assist individuals and families to make arrangements for care [19], to involve carers to greater extent [18] and to avoid patients having to travel [18].

Eleven of the studies described the skill mix of the team [14-18,20$24,26]$. The teams included occupational therapists in ten studies [14$18,20-24,26]$, physiotherapists in nine studies [14-16,18,20-22,24,26], therapy assistants in seven studies [15-17,20-22,24], social workers in six studies [14,16,17,20,23,26], nurses in six studies [14-17,20,21], doctors in six studies $[14,18,22,24,26]$, and speech therapists in five studies $[14,18,22,24,26]$. Reablement was described in detail in 11 studies $[14,15,17,18,20-26]$. Therapy sessions were included in all these 11 studies, individual care plan or goal setting in ten studies [14,20,22$25]$, adaptations to the home in six studies [14,20,22-25], help with activities of daily living in five studies $[20,21,23,25,26]$ and information and signposting in one [20].

\section{Comparators}

The comparators were inpatient rehabilitation $[14,17,22,24,26]$, home care $[15,19,20,23,25]$, day hospital rehabilitation $[15,16,23]$ or no intervention (waiting list control) [19]. The comparator in one study was unclear [21].

\section{Context}

Eight studies took place in the UK [15-18,20,22-24], three in Australia [14,19,25], one in New Zealand [21] and one in Sweden [26]. The earlier studies $(\leq 2005)$ are typically on home rehabilitation vs. hospital rehabilitation for people with stroke $[14,17,18,22,24,26]$ or older people [15]. The studies published in 2006 onwards compared reablement with home care $[16,19-21,23]$ and/or with hospital rehabilitation [16].

\section{Design}

The most frequent study design was cost-consequence analysis $(\mathrm{N}=5)[18,22,23,25,26]$, followed by cost-effectiveness analysis [17,1921], cost-minimisation analysis $[14,16,24]$ and cost-utility analysis $[15,20]$. All studies compared the costs and outcomes using individual data; no study used a decision analytic model. As such, the time horizon corresponds to the follow-up of the individual patients, on which the cost and outcome data was based. This was until the end of reablement [23] or between 6 months [14,19] to two years [21].

\section{Effectiveness data}

Ten studies used data obtained in an RCT [14-18,21,22,24-26], whereas two studies used a retrospective cohort design $[19,23]$ and one used a prospective cohort design [20]. The average sample size was 539 individuals (minimum $=81$; maximum $=3,279$ ). The three non-randomised studies used methods to minimise the impact of selection bias via matching [23], via multivariate adjustment in a Cox proportional hazards model [19] and by using a difference-indifferences regression model [20]. However, no study justified the use of a specific method or stated its assumptions.

\section{Outcome measures}

Table 3 summarises the primary outcome measure collected in the studies. Eight studies specified their primary outcome measure upfront: SF-36 [14,27], Barthel score [18,22,24], need for home care [25], quality-adjusted life years (QALYs) [15], the Nottingham Extended Activities for Daily Living [16] and death or institutionalisation at one year [17]. The primary outcome was inferred from the results for the other four studies: need for home care [23], time to institutionalisation $[19,21]$ and EQ-5D [20]. It was not possible to determine the primary outcome measure in von Koch et al (2001) since all collected outcomes were equally reported [26].

Carer outcomes were collected in eight studies [14$17,21,22,24,26,27]$. The measures collected in more than one study were the Short Form 36 [21,27] and the General Health Questionnaire $[15,27]$ and the proportion satisfied with the care received $[27,28]$.

\section{Resource use and costs}

Table 3 also details the type of costs included in each study. All studies included the costs of reablement and its comparator(s). Secondary care costs were included in 12 studies [14-22,24-26], whereas primary care costs were included in 10 studies [14-18,20-22,24,26]. Social care costs in the community were included in 10 studies [14-18,20,21,23-25] and care home costs were included in five studies [15-17,19,21]. Informal care costs were included in four studies $[14,16,17,21]$. The unit costs for informal care were obtained with the proxy good method as the cost of hostel-level residential care [14], the cost of home help [17] and the average wage of a local authority home care worker [16]. Parsons et al (2006) used the home help rate but did not report the value [21]. No study included productivity costs.

\section{Analytic methods}

The cost-effective intervention was the intervention associated with the lowest costs in the cost-minimisation studies $[14,16,24]$. Three 
Citation: Faria R, Kiss N, Aspinal F, Harden M, Weatherly H (2016) Economic Evaluation of Social Care Interventions: Lessons Drawn from a Systematic Review of the Methods Used to Evaluate Reablement. Health Econ Outcome Res Open Access 2: 107. doi: 10.4172/2471-268x/1000107

Page 4 of 6

\begin{tabular}{|l|l|}
\hline \multicolumn{1}{|c|}{ Topic } & \multicolumn{1}{c|}{ Variables } \\
\hline Decision & Research question \\
\hline Intervention & Perspective \\
\hline Comparator & Description \\
\hline Skill-mix \\
\hline Context & Description \\
\hline Design & Country \\
\hline & Publication date \\
\hline Effectiveness data & Type of economic evaluation \\
\hline & Time horizon \\
\hline Analytic approach \\
\hline Study design \\
\hline Sample size \\
\hline Follow-up \\
\hline Assumptions required \\
\hline Resource use and \\
costs & Primary outcome \\
\hline Analytic methods & Secondary outcomes \\
\hline Carer's outcomes \\
\hline Findings & Types of cost included \\
\hline & The assessment of cost-effectiveness \\
\hline Sensitivity analysis \\
\hline Subgroup analysis \\
\hline & Authors' conclusions \\
\hline Methodological uncertainties and areas for future \\
research
\end{tabular}

Table 1: Data extraction.

\begin{tabular}{|c|c|c|c|}
\hline Comparison: & \multicolumn{3}{|c|}{ For whom: } \\
\cline { 2 - 4 } & Stroke patients & Older people & Anyone \\
\hline $\begin{array}{c}\text { Reablement vs } \\
\text { hospital care }\end{array}$ & $\begin{array}{c}\text { Anderson et al [14] } \\
\text { Beech et al [24] } \\
\text { Donnelly et al [22] } \\
\text { von Koch et al [26] } \\
\text { Kalra et al [17] }\end{array}$ & $\begin{array}{c}\text { Marker et al [15] } \\
\text { Roderick et al [18] }\end{array}$ & No studies \\
\hline $\begin{array}{c}\text { Reablement vs } \\
\text { home care }\end{array}$ & No studies & $\begin{array}{c}\text { Lewin et al [25] } \\
\text { McLeod et al [23] } \\
\text { National evaluation } \\
\text { [19] } \\
\text { Parsons et al [21] }\end{array}$ & $\begin{array}{c}\text { Glendenning et } \\
\text { al [20] }\end{array}$ \\
\hline
\end{tabular}

Table 2: The research question. studies compared the incremental cost-effectiveness ratio (ICER) with a cost-effectiveness threshold $[15,19,20]$. Karla et al and Parsons et al chose the cost-effective intervention on the basis of the intervention with the lowest ICER $[17,21]$. No study discussed the opportunity costs associated with choosing a more costly intervention, and how these compare with its benefits. Nine studies conducted sensitivity analysis; all nine conducted univariate sensitivity analysis [14-21,24] and four studies conducted probabilistic sensitivity analysis $[15,17,19,20]$. No study conducted subgroup analysis.

\section{Findings}

Ten studies found reablement cost-effective. Four studies observed no significant differences in its effectiveness and that reablement is cost-saving $[14,15,24,26]$. Two studies found reablement more effective and cost-saving [22,25]. Four studies found reablement more effective and more costly; of these, two studies considered the additional costs to be reasonable due to potential longer term savings that could not be captured within the time horizon of the analysis $[19,23]$. Parker et al and Roderick et al were unable to conclude on the cost-effectiveness of the intervention because there were no significant differences in the costs and outcomes $[16,18]$.

The studies reported some common areas of methodological uncertainty and future research requirements. Six studies discussed the uncertainty around the impact on carers [14,17,20,22,24,26,28]. Six studies discussed the potential benefits of subgroup analysis to determine how the individual's characteristics affect the costs and outcomes and to tailor reablement to the individual's needs [14,1922,25]. Four studies discussed that more research is required in the appropriate outcome measures $[16,17,19,20]$. The uncertainty in the cost savings from reduced hospital stays was discussed in two studies $[15,19]$. Two studies discussed that more research is required on the cost-effectiveness of different service models $[19,20]$.

\section{Discussion}

The costs and outcomes of reablement have been compared with hospital rehabilitation or home care, first in stroke patients and currently in elderly people. Reablement was shown to be costeffective. Its objectives, team skill-mix and the activities that constitute reablement varied between studies. No study evaluated the contribution of each professional in the team skill-mix and on the different models

\begin{tabular}{|c|c|c|c|c|c|c|c|}
\hline \multirow[t]{2}{*}{ Study } & \multirow[t]{2}{*}{ Outcomes } & \multicolumn{6}{|c|}{ Types of costs } \\
\hline & & Primary care & Secondary care & Social care in community & Residential care & Private & Informal care \\
\hline Anderson et al [27] & SF-36 & $\nabla$ & $\nabla$ & $\nabla$ & & $\nabla$ & $\nabla$ \\
\hline Beech et al [24] & Barthel score & $\nabla$ & $\nabla$ & $\nabla$ & & & \\
\hline Donnelly et al [22] & Barthel score & $\nabla$ & $\nabla$ & & & & \\
\hline Glendenning et al [20] & EQ-5D & $\square$ & $\nabla$ & $\nabla$ & & & \\
\hline Kalra et al [17] & Days at home & $\nabla$ & $\nabla$ & $\nabla$ & $\nabla$ & & $\nabla$ \\
\hline Lewin et al [25] & Need for home care & & $\nabla$ & $\nabla$ & & & \\
\hline McLeod et al [23] & Need for home care & & & $\nabla$ & & & \\
\hline Miller et al [15] & QALYs & $\nabla$ & $\square$ & $\nabla$ & $\nabla$ & & \\
\hline $\begin{array}{l}\text { National Evaluation } \\
\text { [19] }\end{array}$ & Days at home & & $\nabla$ & & $\nabla$ & & \\
\hline Parker et al [16] & ADL/NEADL & $\nabla$ & $\nabla$ & $\nabla$ & $\nabla$ & $\nabla$ & $\nabla$ \\
\hline Parsons et al [21] & Days at home & $\square$ & $\square$ & $\nabla$ & $\nabla$ & $\nabla$ & $\nabla$ \\
\hline Roderick et al [18] & Barthel score & $\nabla$ & $\nabla$ & $\nabla$ & & & \\
\hline von Koch et al [26] & No primary outcome & $\nabla$ & $\nabla$ & & & $\nabla$ & \\
\hline
\end{tabular}

Table 3: Outcomes and types of costs. 
Citation: Faria R, Kiss N, Aspinal F, Harden M, Weatherly H (2016) Economic Evaluation of Social Care Interventions: Lessons Drawn from a Systematic Review of the Methods Used to Evaluate Reablement. Health Econ Outcome Res Open Access 2: 107. doi: 10.4172/2471-268x/1000107

of service delivery. More research is needed on the optimal team skillmix and how best to implement reablement in practice.

All studies used only the data on outcomes and costs collected in each individual study and evaluated cost-effectiveness over the data collection follow-up. This is appropriate if (i) each individual study is the sole source of relevant evidence on the effectiveness and cost of the intervention and comparators, (ii) it includes all relevant comparators and (iii) it follows individuals over the appropriate time horizon. The studies did not discuss these aspects. At least in the subset of reablement for stroke, the range of interventions suggests that there is a variety of ways to deliver reablement that could be evaluated headto-head. Furthermore, reablement can have long term consequences in outcomes and costs, an issue discussed in some studies [19,23], hence a longer time horizon than the study follow-up may be appropriate. Future economic evaluations should consider all the available evidence on the research question, including the different interventions that can be compared, the relevant comparators (e.g., home care, longer hospital stay) and the appropriate time horizon.

Studies using non-randomised data are at risk of selection bias. Selection bias occurs when the outcomes observed in a study are caused by other factors, which are distributed differently across the different patient groups [29]. Selection bias was subject to little discussion and the methods used to address it were not appropriately justified. Future reports should bear in mind that careful analysis and adequate reporting is required when conducting evaluations using non-randomised data to maximise the utility of findings in practice [30].

The outcome measures varied across the studies and some authors expressed concern about whether the outcome measures used were sensitive to the impact of reablement. Furthermore, some studies noted that reablement appeared to have detrimental impact on carers despite a positive effect on individuals. This raises challenges on how to trade-off outcomes in individuals versus their careers and how to include both in an economic evaluation. More research is required on the appropriate outcomes to capture the impact of reablement on both individual users and their careers.

The costs included varied by study, and mostly reflected the perspective of the analysis. There was some variation in the costing of informal care, which reflects the lack of consensus in the literature on this topic [31,32]. Clearer guidance is needed on how to cost informal care.

The assessment of whether reablement or its comparators is cost-effective reveals some variation in the interpretation of the cost-effectiveness decision rules. In principle, the cost-effective intervention offers the most net benefits, that is, its added benefits minus its opportunity costs (i.e., the benefits forgone from displaced interventions to release resources to fund the intervention) [33]. This is straightforward when the intervention generates savings and offers greater or the same benefits $[14,15,22,24-26]$. The challenge is when the intervention generates benefits but is also more costly [17,19-21,23]. The confusion about ICER decision rules revealed in some studies highlights the need to better understand the implications and the use of cost-effectiveness results.

The assessment of cost-effectiveness is made more difficult when interventions have costs and benefits in different sectors (i.e., crosssectoral intervention). Although economic evaluations commonly assume a single budget (e.g., health and social care) and a shared objective, local government, the health care system, individuals and their carers face different budget constraints and have multiple objectives. Hence, the opportunity cost in terms of the benefits forgone vary depending on where the additional costs fall [34]. This is common to many complex interventions that interact between health, social care and the private sphere. Further research is required on how best to handle the implications of cross-sectoral interventions.

No subgroup analysis was conducted but some studies suggested it as an area for future research. Subgroup analysis aims to identify the individuals for whom an intervention is more or less cost-effective so that the intervention will not be offered for subgroups in which it is not cost-effective and will be targeted to those where it is [35]. Future economic evaluations should consider including subgroup analysis in order to better target interventions and resources.

\section{Recommendations for future research}

Future economic evaluations on reablement should include all available evidence relevant to the research question; this includes differences in how reablement is delivered, relevant comparators (e.g., home care, longer hospital stay), and the appropriate time horizon to reflect changes in costs and outcomes. The assessment of costeffectiveness should compare the added benefits to the opportunity costs, and discuss the different sectors affected by the intervention and comparators. Moreover, future evaluations should consider conducting subgroup analysis in order to identify the groups of individuals most likely to benefit from reablement and therefore better target interventions and resources.

More methodological research and guidance is needed on standardised outcome measures for interventions to improve general wellbeing. Some outcome measures have been proposed recently (e.g., Adult Social Care Outcomes Toolkit [36]), but their use in applied evaluations is rare and their sensitivity to different interventions is unclear. Furthermore, more research is needed on outcome measures and/or costing methods to capture the impact of interventions on informal carers, and how to trade off benefits in service users with additional carer burden. This relates to the challenge in assessing cost-effectiveness in cross-sectoral interventions. Some research has proposed methods to account for the trade-offs between the different sectors [34]. However, more work is required on the practical implications for applied economic evaluations.

\section{Strengths and limitations}

This is the first systematic review of the economic evidence on reablement. The search strategy was comprehensive and the inclusion criteria broad. This methodology ensured that a large number of relevant studies were identified and included. Although only 13 studies were identified, it compares positively with a recent systematic review which identified no studies on the effectiveness of reablement [37]. In addition to a full systematic review, this study identified areas for future research and made recommendations for future evaluations.

Reablement is a relatively new term for a group of interventions that has existed for some time; other studies, not identified in the review, might have used terms not included in the search strategy to describe the intervention. However, the purpose of the review was not to ascertain the cost-effectiveness of reablement but to identify the key uncertainties and methods issues, and make recommendations for future research. The issues identified were common to the majority of the studies and highlight the areas where more research is required.

\section{Conclusion}

Reablement has been shown to be cost-effective; however there is 
Citation: Faria R, Kiss N, Aspinal F, Harden M, Weatherly H (2016) Economic Evaluation of Social Care Interventions: Lessons Drawn from a Systematic Review of the Methods Used to Evaluate Reablement. Health Econ Outcome Res Open Access 2: 107. doi: 10.4172/2471-268x/1000107

Page 6 of 6

uncertainty on its costs and benefits in the long term, between different models of service delivery and on the characteristics of the individuals most likely to benefit. Further methodological research is required on the appropriate outcome measures, on how to assess the impact on informal care and on the assessment of value for money in crosssectoral interventions.

\section{Acknowledgement}

The authors are grateful to the MORE project team (Rachel Mann, Bryony Beresford, Gillian Parker, Parvaneh Rabiee, Mona Kanaan, Alison Laver-Fawcett and Gerald Pilkington) for useful discussions on the search strategies and inclusion and exclusion criteria. In addition, the authors are grateful to Andrea Manca for his support during this work.

This project was funded by the National Institute for Health Research Health Services and Delivery Research (NIHR HS\&DR) programme (project number 13/01/17). The views and opinions expressed therein are those of the authors and do not necessarily reflect those of the HS\&DR, NIHR, the UK National Health Service or the Department of Health.

Key: SF-36: Short-form 36 items. ADL: Activities of daily living. NEADL: Nottingham extended activities of daily living. QALYs: Quality-adjusted life years. EQ-5D: Euroqol

\section{References}

1. Parker G (2014) Intermediate Care, Reablement or Something Else? A Research Note about the Challenges of Defining Services.

2. Craig P, Dieppe P, Macintyre S, Michie S, Nazareth I (2008) Developing and evaluating complex interventions: the new Medical Research Council guidance. Bmj 337

3. National Audit Office (2014) Adult social care in England: overview. London Department of Health and Department for Communities and Local Government.

4. National Institute for Health and Care Excellence (2015) Intermediate care including reablement.

5. Parsons JG, Parsons MJ (2012) The effect of a designated tool on person centred goal identification and service planning among older people receiving homecare in New Zealand. Health Soc Care Community 20: 653-662.

6. Lewin GF, Alfonso HS, Alan JJ (2013) Evidence for the long term cost effectiveness of home care reablement programs. Clinical interventions in aging 8:1273.

7. Tuntland H, Espehaug B, Forland O, Hole AD, Kjerstad E, et al. (2014) Reablement in community-dwelling adults: study protocol for a randomised controlled trial. BMC geriatrics 14: 139.

8. Health Workforce Advisory Committee (2006) Care and Support in the Community Setting. Wellington: Health Workforce Advisory Committee.

9. Living well at home: CHSP Good Practice Guide (2015) Australian Government Department of Social Services.

10. Department of Health (2010) The operating framework for the NHS in England 2011/12. London: Department of Health.

11. Systematic Reviews (2009) CRD's guidance for undertaking reviews in health care. York: University of York

12. Rutter D, Francis J, Coren E, Fisher M (2010) SCIE systematic research reviews: guidelines. London: Social Care Institute for Excellence.

13. Centre for Reviews and Dissemination (2015) Search strategies for NHS EED

14. Anderson C, Mhurchu CN, Rubenach S, Clark M, Spencer C, et al. (2000) Home or hospital for stroke Rehabilitation? Results of a randomized controlled trial: Il: cost minimization analysis at 6 months. Stroke 31: 1032-1037.

15. Miller P, Gladman JR, Cunliffe AL, Husbands SL, Dewey ME, et al. (2005) Economic analysis of an early discharge rehabilitation service for older people. Age and Ageing 34: 274-280.

16. Parker SG, Oliver P, Pennington M, Bond J, Jagger C, et al. (2009) Rehabilitation of older patients: day hospital compared with rehabilitation at home - a randomised controlled trial. Health Technology Assessment 13: $1-168$

17. Kalra L, Evans A, Perez I, Knapp M, Swift C, et al. (2005) A randomised controlled comparison of alternative strategies in stroke care. Health Technol
Assess 9: 1-79.

18. Roderick P, Low J, Day R, Peasgood T, Mullee MA, et al. (2001) Stroke rehabilitation after hospital discharge: a randomized trial comparing domiciliary and day-hospital care. Age and Ageing 30: 303-310.

19. National Evaluation of the Transition Care Program RFT206/0506 Final Evaluation Report (2008) Adelaide: Flinders Consulting.

20. Glendenning C, Jones K, Baxter K, Rabiee P, Curtis LA, et al. (2010) Home Care Re-ablement Services: Investigating the longer-term impacts (prospective longitudinal study).

21. Parsons M, Anderson C, Senior H, Chen X, Kerse N, et al. (2006) ASPIRE: Assessment of Services Promoting Independence and Recovery in Elders.

22. Donnelly M, Power M, Russell M, Fullerton K (2004) Randomized controlled trial of an early discharge rehabilitation service: the Belfast community stroke trial. Stroke 35: 127-133.

23. McLeod B, Mair M (2009) Evaluation of City of Edinburgh council home cares re-ablement service. Edinburgh: Scotland. Scottish Government Socia Research.

24. Beech R, Rudd AG, Tilling K, Wolfe CD (1999) Economic consequences of early inpatient discharge to community-based rehabilitation for stroke in an inner-London teaching hospital. Stroke 30: 729-735.

25. Lewin G, Allan J, Patterson C, Knuiman M, Boldy D, et al. (2014) A comparison of the home-care and healthcare service use and costs of older Australians randomised to receive a restorative or a conventional home-care service. Health Soc Care Community 22: 328-336.

26. von Koch L, de Pedro-Cuesta J, Kostulas V, Almazan J, Widen Holmqvist L (2001) Randomized controlled trial of rehabilitation at home after stroke: oneyear follow-up of patient outcome, resource use and cost. Cerebrovascular Diseases 12: 131-138

27. Anderson C, Rubenach S, Mhurchu CN, Clark M, Spencer C, et al. (2000) Home or hospital for stroke rehabilitation? results of a randomized controlled trial: I: health outcomes at 6 months. Stroke 31: 1024-1031.

28. Rudd AG, Wolfe CD, Tilling K, Beech R (1997) Randomised controlled trial to evaluate early discharge scheme for patients with stroke. BMJ 315: 1039-1044.

29. Deeks JJ, Dinnes J, D'amico R, Sowden A, Sakarovitch C, et al. (2003) Evaluating non-randomised intervention studies. Health technology assessment 7: 1-179.

30. Faria R, Hernandez Alava M, Manca A, Wailoo AJ (2015) NICE DSU Technical Support Document 17: The use of observational data to inform estimates of treatment effectiveness for Technology Appraisal: Methods for comparative individual patient data.: NICE DSU.

31. Weatherly H, Faria R, Van den Berg B (2014) Valuing Informal Care for Economic Evaluation. In: Culyer AJ, editor. Encyclopaedia of Health Economics: Elsevier

32. NICE (2015) Developing NICE guidelines: the manual. London: NICE.

33. Drummond MF, Sculpher M, Claxton K, Stoddart GL, Torrance GW (2015) Methods for the economic evaluation of health care programmes.

34. Claxton K, Walker S, Palmer S, Sculpher M (2010) Appropriate Perspectives for Health Care Decisions.

35. Sculpher M (2008) Subgroups and heterogeneity in cost-effectiveness analysis Pharmacoeconomics 26: 799-806.

36. Netten A, Burge P, Malley J, Potoglou D, Towers A-M, et al. (2012) Outcomes of social care for adults: developing a preference-weighted measure. Health Technology Assessment 16.

37. Legg L, Gladman J, Drummond A, Davidson A (2015) A systematic review of the evidence on home care reablement services. Clinical rehabilitation. 